\title{
RENDIMIENTO Y CALIDAD NUTRIMENTAL DE FRIJOL EJOTERO EN DOS AMBIENTES
}

\author{
YIELD AND NUTRITIONAL QUALITY OF SNAP BEAN IN TWO ENVIRONMENTS
}

\author{
Nicolás Salinas Ramírez ${ }^{1}$, J. Alberto Escalante Estrada ${ }^{1 \star}$, Ma. Teresa Rodríguez González ${ }^{1}$ \\ y Eliseo Sosa Montes ${ }^{2}$
}

${ }^{1}$ Postgrado en Botánica, Colegio de Postgraduados, Campus Montecillos. Km. 36.5 carretera México-Texcoco. 56230, Montecillo, Texcoco, Edo. de México. Tel. 01(595) 9520200 ext.1330, Fax 01(595) 95202 30. ${ }^{2}$ Departamento de Zootecnia, Universidad Autónoma Chapingo. Km. 38.5 carretera México-Texcoco. 56235, Chapingo, Estado de México.

*Autor para correspondencia (jasee@colpos.mx)

\section{RESUMEN}

En México existe la cultura y tradición para el consumo de frijol en grano (Phaseolus vulgaris L.), no así para el consumo de ejote (vaina fresca de frijol), esto pese a su alto contenido de proteínas, carbohidratos, fibras, calcio y vitaminas. En el Estado de México, la producción de frijol ejotero no satisface la demanda interna de ejote $(0.9$ a $1.1 \mathrm{~kg}$ per capita), por lo que es necesario importarlo. El bajo rendimiento y calidad nutrimental del ejote puede deberse a la falta de manejo apropiado del cultivo y a la falta de adaptación de las variedades utilizadas. Por ello, el presente estudio se hizo para dilucidar la naturaleza de esta interacción en frijol ejotero. Se compararon tres variedades: 'Strike' y 'Black Valentine' de crecimiento determinado, y 'Hav-14' de crecimiento indeterminado, en dos ambientes contrastantes (Montecillo, de clima semiárido; y San Pablo Ixayoc, de clima templado) en el Estado de México. La siembra se hizo el 7 de mayo de 2008, bajo régimen de lluvia estacional. El diseño experimental fue de bloques completos al azar, en cada ambiente. Se registraron las variables: días a eventos fenológicos, rendimiento en ejote (peso fresco, $\mathrm{g} \mathrm{m}^{-2}$ ), número de ejotes $/ \mathrm{m}^{2}, \mathrm{y}$ calidad nutricional. En los ambientes de prueba se colectaron datos de temperaturas máxima y mínima, precipitación, y se calculó la evapotranspiración (ETc) y las unidades calor (UC). El tiempo de ocurrencia de las etapas fenológicas del frijol fue diferente entre cultivares y ambientes. En Montecillo, 'Strike' presentó el ciclo más corto $(78 \mathrm{~d}$ de siembra a último corte) y 'Hav-14' el más largo (102 d). En San Pablo 'Strike' alargó su ciclo en 12 d, y 'Hav-14' en 17 d. En Montecillo el rendimiento y número de ejotes más altos $\left(1.37 \mathrm{~kg} \mathrm{~m}^{-2}\right.$ y 307$)$ correspondieron al $\mathrm{cv}$. 'Hav-14' con 782 UC, $175 \mathrm{~mm}$ de ETc y $275 \mathrm{~mm}$ de precipitación, y los más bajos al cv. 'Strike' ( $0.195 \mathrm{~kg} \mathrm{~m}^{-2}$ y 57 ejotes, respectivamente) en San Pablo (567 UC, 151 ETc y 379 mm de precipitación). La calidad nutrimental del ejote presentó diferencias entre ambientes; la var. 'Hav-14' registró los contenidos más altos en proteína $(22.3 \%)$, minerales $(8.8$ \%) y fibra (ácida-FDA- $24.3 \%$ y neutra -FDN- $31.5 \%$ ) en Montecillo. En contraste, en San Pablo esta variedad mostró una tendencia opuesta, con los valores más bajos en proteína (18\%), minerales (6.1 \%), FDA $(17.4 \%)$ y FDN $(24.4 \%)$. La interacción genotipo $x$ ambiente fue significativa, ya que las variables rendimiento, número de ejotes y calidad nutrimental variaron en función del ambiente de desarrollo.

Palabras clave: Phaseolus vulgaris, fenología, climas, interacción cultivar $\mathrm{x}$ ambiente, rendimiento.

\section{SUMMARY}

In México dry bean (Phaseolus vulgaris L.) is traditional, but not of snap bean (fresh bean pods) despite its high contents of proteins, carbohydrates, fiber, calcium and vitamins. In the State of México, snap bean production does not satisfy the domestic demand, thus requiring importation. Since yield and nutritional quality of the fresh pods may be a function of cultivar and environmental conditions, the aim of this study was to elucidate the nature of this interaction in snap bean production. Three cultivars were tested, determinate growth habit varieties 'Strike' and 'Black Valentine', indeterminate climbing growth habit variety and 'Hav-14', in two contrasting environments (Montecillo, semiarid climate; and San Pablo Ixayoc, temperate climate) in the State of México. Sowing was done on May 7, 2008, under rainfed conditions. The experimental design was a randomized complete block for each environment. Variables recorded were: days to phenologic events, fresh pod weight (in $\mathrm{g} \mathrm{m}^{-2}$, number of pods per $\mathrm{m}^{2}$, and nutritional quality. With regard to the environment, collected data included maximum and minimum temperatures, precipitation; evapotranspiration (ETc); and Heat Units (UC) were calculated. The time of occurrence of the phenologic stages of bean plants varied among cultivars and environments. In Montecillo cv. 'Strike' presented the shortest cycle $(78 \mathrm{~d}$ from sowing to last harvest) and cv. 'Hav-14' the longest (102 d). In San Pablo cv.'Strike' extended its cycle by $12 \mathrm{~d}$ and 'Hav-14' by $17 \mathrm{~d}$. In Montecillo, the highest yield and number of pods $\left(1.37 \mathrm{~kg} \mathrm{~m}^{-2}\right.$ and 307$)$ corresponded to cv.'Hav-14' with $782 \mathrm{UC}, 175 \mathrm{~mm}$ of and ETc and $275 \mathrm{~mm}$ of precipitation, and the lowest values to $\mathrm{cv}$. 'Strike' $\left(0.195 \mathrm{~kg} \mathrm{~m}^{-2}\right.$ and 57 beans, respectively) in San Pablo (567 UC, 151 ETc and $379 \mathrm{~mm}$ of precipitation). The pod nutritional quality showed differences between environments: in Montecillo cv. 'Hav-14' recorded the highest contents of proteins (22.3\%), minerals ( $8.8 \%$ ) and fiber (acid, -ADF- $24.3 \%$, and neutral, -NDF- $31.5 \%$ ); while at San Pablo, the opposite trend was observed, with lowest values in proteins $(18 \%)$, minerals $(6.1 \%)$, ADF $(17.4 \%)$ and NDF (24.4\%). A significant genotype $x$ environment interaction was found, since variables yield, number of beans and nutritional quality varied depending on the test location.

Index words: Phaseolus vulgaris, phenology, yield, climate, interaction cultivar $\mathrm{x}$ environment.

\section{INTRODUCCIÓN}

En México existe cultura y tradición para el consumo de frijol en grano (Phaseolus vulgaris L.), no así para el ejote (vaina fresca de frijol), a pesar de sus altos contenidos de proteínas, carbohidratos, fibras, calcio y vitaminas (Salinas et al., 2008; Quintana et al., 1999; Guzmán et al., 2002). La productividad de frijol ejotero a nivel mundial es de $10.6 \mathrm{t}$ ha $^{-1}$ (Yvestirilly, 2002), y en México se cosechan $10 \mathrm{tha}^{-1}$ en condiciones de riego. Los principales estados productores son: Morelos, Puebla, Hidalgo y Sinaloa, que en conjunto producen $78.9 \%$ del total nacional (SAGARPA, 2009). En el Estado de México se reportan 40 ha con un rendimiento de $3.7 \mathrm{t} \mathrm{ha}^{-1}$, rendimiento que no satisface la demanda interna 
de ejote ( 0.9 a $1.1 \mathrm{~kg}$ per capita), por lo que es necesario importarlo. El bajo rendimiento puede deberse en parte a la falta de un manejo apropiado de las variedades utilizadas y a la falta de adaptación. Para revertir lo anterior se deben diseñar estrategias con prácticas agronómicas que conduzcan al incremento del rendimiento y calidad nutricional del frijol ejotero, como arreglos topológicos, fertilización, fechas de siembra e introducción de nuevos cultivares. En condiciones de clima templado y con riego, Esquivel et al. (2006) encontraron que el frijol ejotero de hábito indeterminado puede rendir $\left(25.6 \mathrm{t} \mathrm{ha}^{-1}\right)$ y los de tipo determinado (22.2 t ha ${ }^{-1}$ ). Según Peixoto et al. (2002), el cv. 'Hav-14' requiere $42 \mathrm{~d}$ de siembra a antesis para un rendimiento de $21.7 \mathrm{t} \mathrm{ha}^{-1}$ bajo condiciones de riego y en clima templado. En clima semiárido y en condiciones de temporal (secano) la floración de 'Hav-14' ocurre a los 60 d con un rendimiento de $11.7 \mathrm{t} \mathrm{ha}^{-1}$ (Salinas et al., 2008).

$\mathrm{Al}$ ejote se le atribuyen propiedades nutracéuticas, por presentar un bajo contenido calórico de $133.9 \mathrm{~kJ}$ por cada $100 \mathrm{~g}$ de fruto fresco (Adsule et al., 2004), que puede ayudar a reducir el sobrepeso y la obesidad. Asimismo, por su alto contenido de fibra $(25 \%)$ reduce el tiempo de tránsito intestinal, la tasa de glucosa sanguínea postprandial, la absorción de grasa y colesterol, y está relacionado también con la prevención del cáncer de colon (Yvestirilly, 2002). La calidad nutricional del ejote varía en función del cultivar. Zhiwei et al. (1995) encontraron que la fibra y proteína cruda de 51 genotipos de frijol ejotero promediaron 11.6 g y 28 g por cada $100 \mathrm{~g}$ de peso seco, respectivamente. En cultivares de crecimiento indeterminado los contenidos de fibra y proteína cruda fueron de $16.2 \mathrm{~g}$ y $30 \mathrm{~g}$ por $100 \mathrm{~g}$ de peso seco (Esquivel et al., 2006). La acumulación de calcio en la vaina está más relacionada con el genotipo que con su disponibilidad en el suelo (Miglioranza et al., 2003; Pomper y Grusac, 2004; Favaro et al., 2007). Algunos autores han reportado que la fenología, el rendimiento y la calidad nutrimental del frijol ejotero podrían estar determinados por el ambiente de producción (Traka et al., 2000; Mawgoud et al., 2005).

Los estudios en frijol ejotero en el país se han efectuado bajo riego, en ambientes de Morelos (clima cálido subhúmedo), Hidalgo (clima seco), Puebla y Tlaxcala (clima templado subhúmedo) con rendimientos promedio de $8.5 \mathrm{t} \mathrm{ha}^{-1}$, superiores a la media reportada para el Estado de México de 3.7 t ha $^{-1}$ (Salinas et al., 2008). Sin embargo, es limitada la información sobre el comportamiento agronómico del frijol ejotero en condiciones de régimen de lluvia estacional para el ambiente de Montecillo (clima semiárido) y San Pablo Ixayoc (clima templado), localidades del Estado de México que tienen, áreas cultivadas con esta especie. El objetivo de este estudio fue determinar la influencia del ambiente sobre el crecimiento, el rendimiento y la calidad nutrimental de tres cultivares de frijol ejotero.

\section{MATERIALES Y MÉTODOS}

El estudio se llevó a cabo en dos ambientes de importancia agrícola de esta región: 1) En Montecillo (19²9’ LN, $98^{\circ} 53^{\prime} \mathrm{LO}$, a $2250 \mathrm{msnm}$ ), cuyo clima es semiárido BS1, el menos seco de los áridos, con lluvias en verano, temperatura media anual de $14.6{ }^{\circ} \mathrm{C}$ y $558.5 \mathrm{~mm}$ de precipitación, en un suelo franco arenoso y pH de 7.0; 2) En San Pablo Ixayoc ( $19^{\circ} 33^{\prime} \mathrm{LN}, 98^{\circ} 47^{\prime} \mathrm{LO}$, a $2600 \mathrm{msnm}$ ), con clima templado subhúmedo C (W0) (w), con lluvias en verano (García, 2005), temperatura media anual de $14.7^{\circ} \mathrm{C}$ y 609 $\mathrm{mm}$ de precipitación, en un suelo migajón arenoso y $\mathrm{pH}$ 6.3. Ambos sitios se ubican en el municipio de Texcoco, Estado de México.

Los tratamientos fueron dos variedades de hábito de crecimiento determinado ('Strike' y 'Black Valentine') y una de crecimiento indeterminado ('Hav-14'), cuya siembra se hizo a mano el 7 de mayo de 2008 en los dos ambientes, a una densidad de 6.25 plantas $\mathrm{m}^{-2}$. Se usó un diseño experimental de bloques al azar, con cuatro repeticiones. La unidad experimental fue de $3.2 \mathrm{~m}$ por $6 \mathrm{~m}$ de longitud (19.2 $\mathrm{m}^{2}$ ). Con una estación meteorológica marca Campbell ${ }^{\circledR}$ (Campbell Scientific Inc., USA) se registraron las variables climáticas: precipitación $(\mathrm{mm})$ y temperaturas máxima y mínima $\left({ }^{\circ} \mathrm{C}\right)$, con las que se calcularon sus promedios semanales durante el ciclo del cultivo. También se calcularon las unidades calor (UC, ${ }^{\circ} \mathrm{Cd}$ ) de acuerdo con Snyder (1985), y la evapotranspiración del cultivo (ETc, en $\mathrm{mm}$ ), como señalaron Doorenbos y Pruitt (1986).

En las plantas se tomaron datos de fenología en cuatro etapas vegetativas $(\mathrm{V}-1=$ emergencia, $\mathrm{V}-2=$ primer par de hojas primarias, $\mathrm{V}-3=$ primer par de hojas trifolioladas, $\mathrm{y}$ $\mathrm{V}-4=$ tercer par de hojas trifolioladas), $\mathrm{y}$ en tres etapas reproductivas $(\mathrm{R}-5=$ prefloración, $\mathrm{R}-6$ = floración, y F-7 = formación de vainas), de acuerdo con la escala descrita por Escalante y Kohashi (1993). Para rendimiento se hicieron siete cortes de ejote con intervalos de $3 \mathrm{~d}$, cuando las vainas alcanzaron una longitud mayor a $10 \mathrm{~cm}$, y en cada corte se registró peso fresco $\left(\mathrm{kg} \mathrm{m}^{-2}\right)$ y número de ejotes $/ \mathrm{m}^{2}$.

La calidad nutrimental de los ejotes (porciento de minerales, calcio, fósforo, carbohidratos solubles, fibra detergente ácida, lignina, fibra detergente neutra, hemicelulosa, proteína y grasa) se determinó mediante un análisis químico proximal (Sosa, 1979), y para calcular la humedad de los frutos éstos se secaron en una estufa de aire forzado (THELCO Modelo $28 \circledR$ ) a $55^{\circ} \mathrm{C}$, hasta peso constante. Las muestras secas se trituraron en un molino eléctrico (Janke and Kunkel Inka Modelo Kb 5/10®, Germany), con criba de $50 \mu \mathrm{m}$. A los datos colectados de cada variable se le hizo 
un análisis de varianza combinado (ambientes por cultivares). Para la comparación de medias se aplicó la prueba de Tukey $(\alpha=0.05)$.

\section{RESULTADOS Y DISCUSIÓN}

\section{Relación de la fenología con la precipitación y temperatura}

Hubo diferencia varietal para el tiempo de ocurrencia de las etapas fenológicas. Las variedades de hábito determinado registraron un promedio de $89 \mathrm{~d}$ de la siembra al último corte, y la de hábito indeterminado duró $110 \mathrm{~d}$ en promedio. En Montecillo, la var. 'Strike' mostró el periodo más corto de siembra a último corte (78 d) y la var. 'Hav-14' el más largo (102 d), como se ilustra en la Figura 1a. Estas dos variedades, 'Strike' y 'Hav-14', mostraron en San Pablo un ciclo reproductivo 12 y $17 \mathrm{~d}$ más prolongado que en Montecillo (Figura 1-b).

Estas diferencias pueden explicarse por una mejor distribución de la precipitación en Montecillo (33 \% en la fase vegetativa y $67 \%$ en la reproductiva). En contraste, en San Pablo la mayor precipitación ocurrió en la fase vegetativa (54\%) y la menor en la reproductiva (46\%). Con respecto a la temperatura, la máxima promedio más alta $\left(25^{\circ} \mathrm{C}\right)$ se registró en Montecillo y la más baja $\left(22^{\circ} \mathrm{C}\right)$ en San Pablo, diferencia que podría explicar el que los cultivares 'Strike' y 'Hav-14' hayan tenido un ciclo más largo en San Pablo. Estos resultados sugieren que la disponibilidad de humedad, la temperatura y su interacción influyen en la fenología del frijol ejotero. Tendencias similares sobre la fenología del frijol para grano fueron reportados por Rosales et al. (2001), ya que encontraron que la reducción en la temperatura máxima durante el ciclo del cultivo retrasó la fenología del mismo.

Rendimiento, acumulación de unidades calor (UC, ${ }^{\circ} \mathrm{Cd}$ ), evapotranspiración del cultivo $(\mathrm{ETc}, \mathrm{mm})$ y precipitación (PP, mm).

El rendimiento promedio de ejote fue de $0.671 \mathrm{~kg} \mathrm{~m}^{-2}$ (Cuadro 1), superior al promedio $0.37 \mathrm{~kg} \mathrm{~m}^{-2}$ reportado para el Estado de México (SAGARPA, 2009). En Montecillo la var. 'Hav-14' produjo mayor rendimiento y mayor número de ejotes, seguida de 'Black Valentine' y de 'Strike'. Se encontraron tendencias similares en San Pablo, donde 'Hav-14' rindió más que 'Black Valentine' y 'Strike'. La diferencia en rendimiento entre cultivares podría relacionarse con diferencias en la duración del ciclo biológico entre hábitos de crecimiento, puesto que los de hábito indeterminado tienen mayor oportunidad de ocupar el espacio y los insumos para su crecimiento y rendimiento; por ejemplo, el cv. 'Hav-14' de hábito de crecimiento indeterminado

Cuadro 1. Rendimiento de frijol ejotero $\left(\mathrm{kg} \mathrm{m}^{-2}\right)$, número de ejotes por $\mathrm{m}^{2}$, variables e índices ambientales en función del cultivar. Montecillo y San Pablo Ixayoc, Edo. de México. Verano 2008.

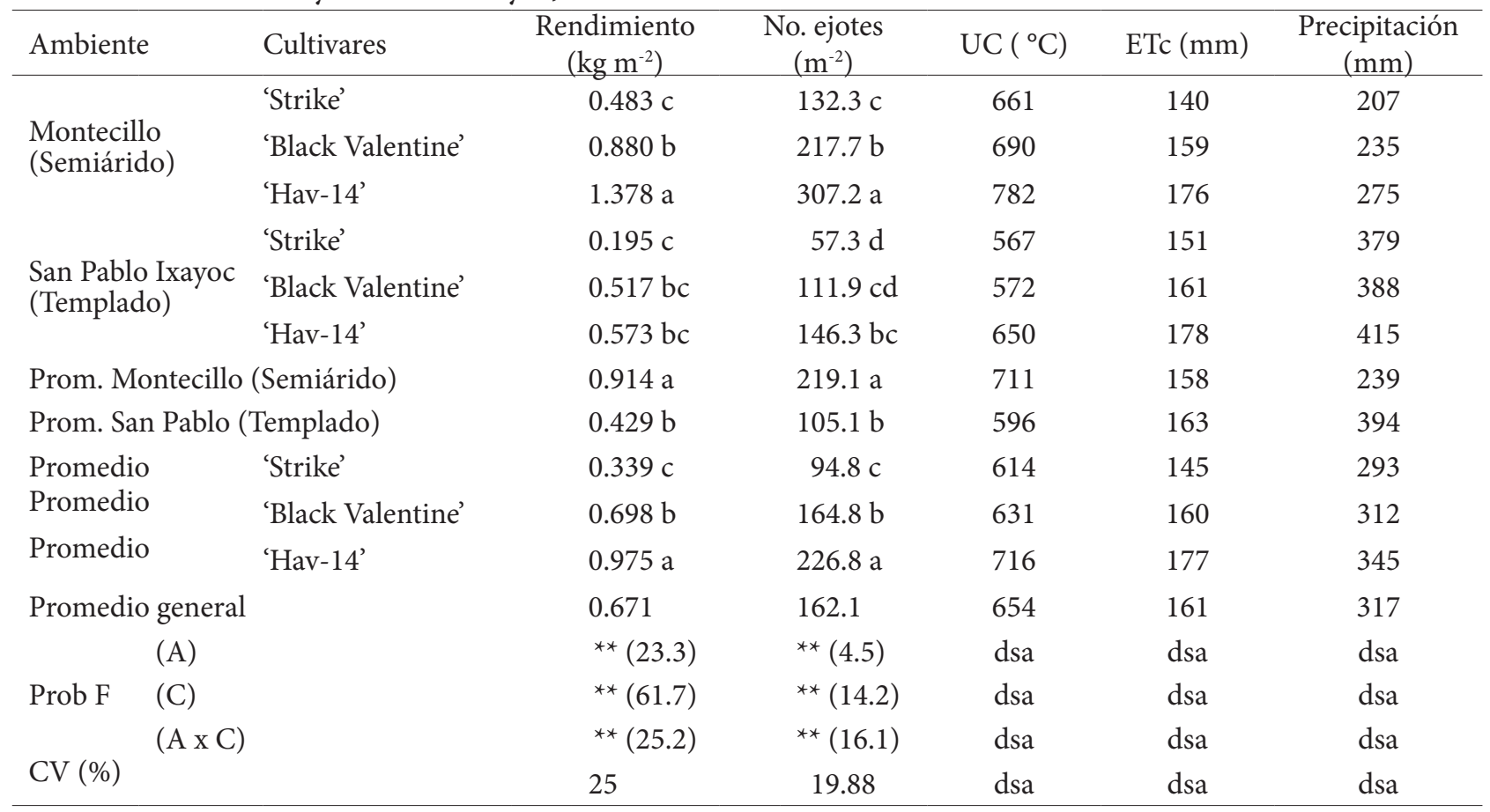

Medias con la misma letra dentro de columnas son estadísticamente iguales (Tukey 0.05). ${ }^{\star \star} \mathrm{P}>0.01 ; \mathrm{UC}=$ unidades calor; ETc $=$ evapotranspiración; $\mathrm{dsa}=$ datos sin analizar; $(=\mathrm{DMS}$ de Tukey a $5 \%$; CV = coeficiente de variación; $\mathrm{A}=$ ambiente; $\mathrm{C}=$ cultivar; $\mathrm{A} \mathrm{x} \mathrm{C}=$ interacción ambiente $\mathrm{x}$ cultivar. 


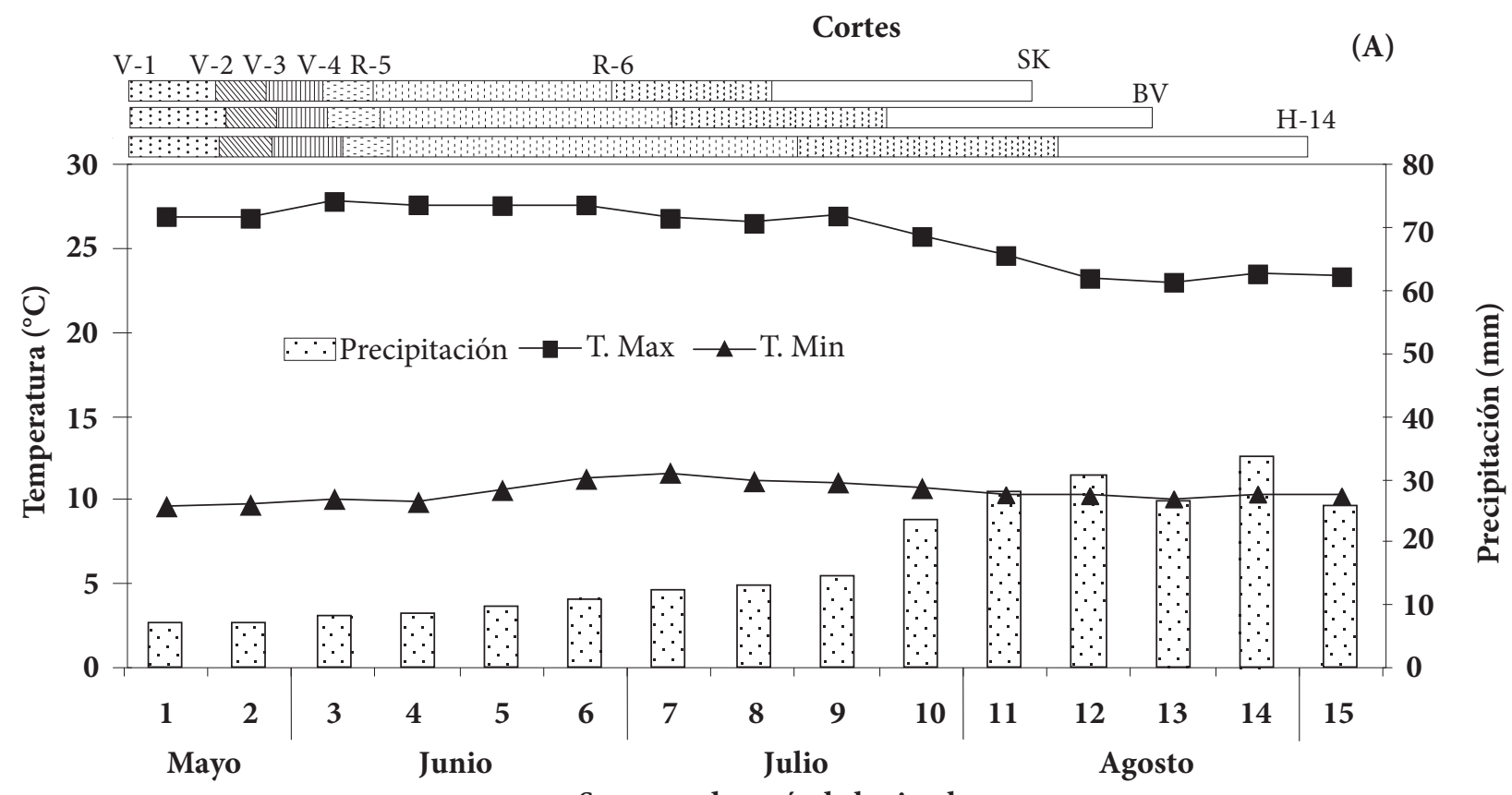

Semanas después de la siembra

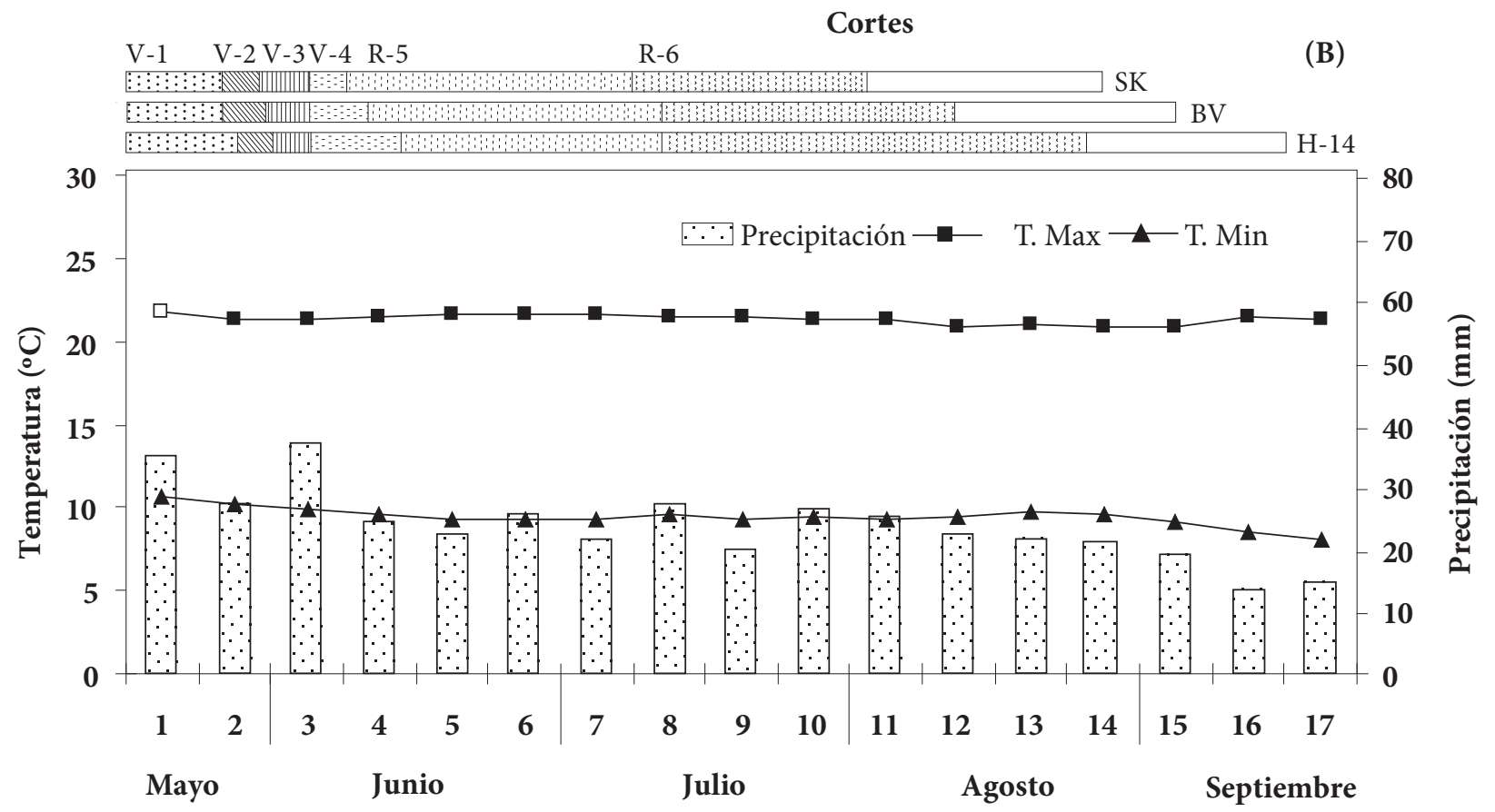

Semanas después de la siembra

Figura 1. Fenología del frijol ejotero (Phaseolus vulgaris L.), medias semanales de temperaturas máxima y mínima, y suma semanal de la precipitación. Clima semiárido (Montecillo, A) y clima templado (San Pablo Ixayoc, B) Estado de México. Verano 2008. Etapas fenológicas del frijol ejotero: V-1 = emergencia; V-2 = hojas primarias; V-3 = primera hoja trifoliolada; $\mathrm{V}-4$ = tercera hoja trifoliolada; $\mathrm{R}-5$ = prefloración; $\mathrm{R}-6$ = floración; $\mathrm{R}-7$ = formación de vainas. Cultivares: SK = 'Strike'; $\mathrm{BV}=$ 'Black Valentine' y H-14 = 'Hav-14'. 
mostró un ciclo más largo y acumuló más UC, ETc y PP, en contraste con el cv. 'Strike' de ciclo corto.

Conforme a esta tendencia, se esperaría que 'Hav-14' rindiera más fruto en San Pablo donde llovió $415 \mathrm{~mm}$ en todo su ciclo, comparado con el Montecillo que recibió $275 \mathrm{~mm}$. Sin embargo, la diferencia en humedad disponible durante la etapa reproductiva entre ambientes ocasionó que en Montecillo 'Hav-14' contara con más agua $(116 \mathrm{~mm})$ del primero al séptimo corte, que en San Pablo donde solo tuvo $69 \mathrm{~mm}$ en el mismo período, lo que explica las diferencias en rendimiento a favor de Montecillo. Tendencias similares fueron observadas por Cselotei y Vargas (1987).

En Montecillo se observó mayor número de ejotes que en San Pablo, lo que confirma que la distribución de la lluvia es un factor determinante para el rendimiento del frijol ejotero. En cuanto a la interacción genotipo ambiente, en 'Hav-14' se observó que las condiciones climáticas de Montecillo favorecieron el rendimiento y número de ejotes de este cultivar, en contraste con las condiciones de San Pablo en donde se registraron los valores más bajos (Cuadro 1).

Estos resultados indican que las condiciones de lluvia en Montecillo fueron más favorables para una mayor expresión del crecimiento y rendimiento del cv. 'Hav-14'. En cuanto a las temperaturas mínima y máxima óptimas (15 ${ }^{\circ} \mathrm{C}$ y $27^{\circ} \mathrm{C}$ ) y pH del suelo (5.5 a 6.5) para crecimiento y rendimiento de frijol de grano, Ríos y Quirós (2007) encontraron que las condiciones de los sitios de estudio fueron limitantes para la expresión del crecimiento y rendimiento del frijol ejotero, puesto que la temperatura mínima osciló entre 9 y $10^{\circ} \mathrm{C}$ y la máxima entre 22 a $27^{\circ} \mathrm{C}$ (Figura 1 ).

\section{Calidad nutrimental de frutos en cultivares de frijol ejotero}

Según el análisis proximal de los cvs. 'Strike' y 'Hav-14', su contenido nutrimental mostró efectos significativos del ambiente de prueba. Los valores más altos de proteína, minerales, lignina y FDA se encontraron en Montecillo. En cambio, los porcentajes de grasa, hemicelulosa, fósforo y calcio no presentaron diferencias entre localidades. En promedio, por cada $100 \mathrm{~g}$ de materia seca el frijol ejotero aporta $7.8 \%$ de minerales, $1.4 \%$ de calcio, $0.06 \%$ de fósforo, 1.4 $\%$ de grasa, $44 \%$ de carbohidratos solubles, $20.6 \%$ de fibra detergente ácida (FDA), $27.1 \%$ de fibra detergente neutra (FDN) y $19.7 \%$ de proteínas. Estos valores indican que el ejote es un alimento con bajo contenido calórico $(133.9 \mathrm{~kJ}$ por $100 \mathrm{~g}$ de materia seca) y alta calidad proteica, que lo hace una alternativa para incrementar su consumo y contribuir a reducir los problemas de sobrepeso y obesidad que actualmente afectan a más de la mitad de la población en México (Secretaría de Salud, 2010).

Según Miglioranza et al. (2003), el contenido de calcio en el ejote depende principalmente del cultivar utilizado y no de la disponibilidad de este mineral en el suelo. No obstante, en Montecillo el cv.'Hav-14' mostró los contenidos más altos en proteína, minerales, FDA y FDN $(22.3 \%, 8.8 \%$, $24.3 \%$ y $31.5 \%$, respectivamente), en comparación con los valores registrados en San Pablo (17.9 \%, 6.1 \%, 17.4 \%, 24.4 $\%$, respectivamente), como se muestra en el Cuadro 2. Dichas diferencias pueden ser explicadas por la variación ambiental entre las dos localidades, principalmente la distribución de la precipitación durante las etapas reproductivas del cultivo (prefloración, floración y formación de vaina).

Al comparar el contenido nutrimental promedio de cada ambiente, en Montecillo se obtuvieron los porcentajes más altos en minerales (8.6\%), fósforo (0.06 \%), FDA (21.9\%), lignina (0.2\%), FDN (28.5\%) y proteína (20.3\%), mientras que en San Pablo se obtuvieron los porcentajes más altos de calcio (1.4 \%), carbohidratos solubles $(46.5 \%)$ y grasa (1.6 $\%)$. Entre los cultivares, 'Hav-14' presentó los valores más altos en calcio (1.5\%), fósforo (0.07\%), proteína (20.1\%) y grasa (1.7\%), mientras que los más bajos ocurrieron en 'Strike' ( $1.2 \%, 0.06 \%, 19.0 \%, 1.0 \%$, respectivamente).

La interacción genotipo $\mathrm{x}$ ambiente resultó significativa, lo que demuestra que el ambiente tiene un efecto directo en la expresión nutrimental de los cultivares. Por ejemplo, en 'Hav-14' las condiciones del clima de Montecillo fueron favorables para que acumulara mayores contenidos de minerales, lignina y proteína (Cuadro 2) que en San Pablo (clima templado). Estos resultados sugieren la necesidad de continuar los estudios sobre el comportamiento de los cultivares en ambientes específicos, para encontrar las condiciones de desarrollo más apropiadas que produzcan mayor rendimiento y calidad nutrimental.

\section{CONCLUSIONES}

Los eventos fenológicos, el rendimiento y la calidad nutrimental del frijol ejotero están en función del cultivar y del ambiente. En Montecillo (clima semiárido) y San Pablo (clima templado) el cv. 'Hav-14' de hábito de crecimiento indeterminado presentó la mayor producción de ejote, y en Montecillo dio la mejor calidad nutrimental. La existencia de interacción genotipo $\mathrm{x}$ ambiente indica que los cultivares evaluados responden de manera diferente al ambiente de producción en cuanto a su rendimiento, número de ejotes y calidad nutrimental. 
Cuadro 2. Análisis químico proximal (\%), de cultivares de frijol ejotero de diferente hábito de crecimiento crecidos en Montecillo (clima semiárido) y San Pablo Ixayoc (clima templado), Estado de México. 2008.

\begin{tabular}{|c|c|c|c|c|c|c|}
\hline \multirow{2}{*}{\multicolumn{2}{|c|}{ Ambiente/cultivar }} & \multicolumn{5}{|c|}{ Nutrimentos } \\
\hline & & Minerales & Calcio & Fósforo & CS & FDA \\
\hline \multicolumn{2}{|c|}{ Mont SK } & $7.9 \mathrm{~b}$ & $1.2 \mathrm{c}$ & $0.07 \mathrm{a}$ & $42.9 \mathrm{c}$ & $22.4 \mathrm{ab}$ \\
\hline \multicolumn{2}{|c|}{ Mont BV } & $9.1 \mathrm{a}$ & $1.4 \mathrm{~b}$ & $0.05 \mathrm{~d}$ & $45.9 \mathrm{~b}$ & $18.9 \mathrm{c}$ \\
\hline \multicolumn{2}{|c|}{ Mont H-14 } & $8.8 \mathrm{a}$ & $1.5 \mathrm{ab}$ & $0.07 \mathrm{a}$ & $35.7 \mathrm{~d}$ & $24.3 \mathrm{a}$ \\
\hline \multicolumn{2}{|c|}{ SPI SK } & $7.3 \mathrm{c}$ & $1.2 \mathrm{c}$ & $0.06 \mathrm{c}$ & $44.3 \mathrm{bc}$ & $21.7 \mathrm{~b}$ \\
\hline \multicolumn{2}{|l|}{ SPI BV } & $7.4 \mathrm{c}$ & $1.5 \mathrm{a}$ & $0.06 \mathrm{~b}$ & $45.6 \mathrm{~b}$ & $19.3 \mathrm{c}$ \\
\hline \multicolumn{2}{|c|}{ SPI H-14 } & $6.1 \mathrm{~d}$ & $1.5 \mathrm{ab}$ & $0.07 \mathrm{a}$ & $49.7 \mathrm{a}$ & $17.4 \mathrm{c}$ \\
\hline \multicolumn{2}{|c|}{ Prom. Mont } & $8.6 \mathrm{a}$ & $1.3 \mathrm{~b}$ & $0.06 \mathrm{a}$ & $41.5 \mathrm{~b}$ & $21.9 \mathrm{a}$ \\
\hline \multicolumn{2}{|c|}{ Prom. SPI } & $6.9 \mathrm{~b}$ & $1.4 \mathrm{a}$ & $0.06 \mathrm{a}$ & $46.5 \mathrm{a}$ & $19.4 \mathrm{~b}$ \\
\hline \multicolumn{2}{|c|}{ Prom. SK } & $7.6 \mathrm{~b}$ & $1.2 \mathrm{~b}$ & $0.06 \mathrm{~b}$ & $43.6 \mathrm{~b}$ & $22.0 \mathrm{a}$ \\
\hline \multicolumn{2}{|c|}{ Prom. BV } & $8.2 \mathrm{a}$ & $1.5 \mathrm{a}$ & $0.05 \mathrm{c}$ & $45.7 \mathrm{a}$ & $19.1 \mathrm{c}$ \\
\hline \multicolumn{2}{|c|}{ Prom. H-14 } & $7.5 \mathrm{~b}$ & $1.5 \mathrm{a}$ & $0.07 \mathrm{a}$ & $42.7 \mathrm{~b}$ & $20.8 \mathrm{~b}$ \\
\hline \multicolumn{2}{|c|}{ General } & 7.8 & 1.4 & 0.06 & 44 & 20.6 \\
\hline \multirow{3}{*}{ Prob F } & A & $* *(0.13)$ & $* *(0.04)$ & ns & $\star *(0.73)$ & $\star *(0.71)$ \\
\hline & $\mathrm{C}$ & $* *(0.37)$ & $* *(0.11)$ & $* *(0.01)$ & $\star *(1.78)$ & ** $(1.93)$ \\
\hline & $A \times C$ & $* *(0.18)$ & $* *(0.9)$ & $* *(0.04)$ & $\star *(0.84)$ & $* *(1.02)$ \\
\hline \multirow{2}{*}{\multicolumn{2}{|c|}{ CV (\%) }} & 1.70 & 2.99 & 1.78 & 1.59 & 3.29 \\
\hline & & Lignina & FDN & Hemicelulosa & Proteína & Grasa \\
\hline \multicolumn{2}{|c|}{ Mont SK } & $0.3 \mathrm{a}$ & $30.7 \mathrm{a}$ & $8.3 \mathrm{a}$ & $18 \mathrm{~d}$ & $0.4 \mathrm{~d}$ \\
\hline \multicolumn{2}{|c|}{ Mont BV } & $0.1 \mathrm{~b}$ & $23.2 \mathrm{c}$ & $4.21 \mathrm{c}$ & $20.7 \mathrm{~b}$ & $1.2 \mathrm{c}$ \\
\hline \multicolumn{2}{|c|}{ Mont H-14 } & $0.3 \mathrm{a}$ & $31.5 \mathrm{a}$ & $7.1 \mathrm{ab}$ & $22.3 \mathrm{a}$ & $1.8 \mathrm{a}$ \\
\hline \multicolumn{2}{|c|}{ SPI SK } & $0.1 \mathrm{~b}$ & $26.5 \mathrm{~b}$ & $4.8 \mathrm{bc}$ & $20.1 \mathrm{~b}$ & $1.7 \mathrm{a}$ \\
\hline \multicolumn{2}{|l|}{ SPI BV } & $0.1 \mathrm{~b}$ & $26.5 \mathrm{~b}$ & $7.3 \mathrm{ab}$ & $18.9 \mathrm{c}$ & $1.5 \mathrm{~b}$ \\
\hline \multicolumn{2}{|c|}{ SPI H-14 } & $0.1 \mathrm{~b}$ & $24.4 \mathrm{c}$ & $7.1 \mathrm{ab}$ & $17.9 \mathrm{~d}$ & $1.7 \mathrm{a}$ \\
\hline \multicolumn{2}{|c|}{ Prom. Mont } & $0.2 \mathrm{a}$ & $28.5 \mathrm{a}$ & $6.5 \mathrm{a}$ & $20.3 \mathrm{a}$ & $1.1 \mathrm{~b}$ \\
\hline \multicolumn{2}{|c|}{ Prom. SPI } & $0.1 \mathrm{~b}$ & $25.8 \mathrm{~b}$ & $6.4 \mathrm{a}$ & $18.9 \mathrm{~b}$ & $1.6 \mathrm{a}$ \\
\hline \multicolumn{2}{|c|}{ Prom. SK } & $0.2 \mathrm{a}$ & $28.6 \mathrm{a}$ & $6.5 \mathrm{a}$ & $19.0 \mathrm{~b}$ & $1.0 \mathrm{c}$ \\
\hline \multicolumn{2}{|c|}{ Prom. BV } & $0.1 \mathrm{~b}$ & $24.8 \mathrm{~b}$ & $5.7 \mathrm{a}$ & $19.8 \mathrm{a}$ & $1.3 \mathrm{~b}$ \\
\hline \multicolumn{2}{|c|}{ Prom. H-14 } & $0.2 \mathrm{a}$ & $28 \mathrm{a}$ & $7.1 \mathrm{a}$ & $20.1 \mathrm{a}$ & $1.7 \mathrm{a}$ \\
\hline \multicolumn{2}{|c|}{ General } & 0.2 & 27.1 & 6.5 & 19.6 & 1.4 \\
\hline & A & $\star *(0.07)$ & $\star *(0.59)$ & NS & $* *(0.31)$ & $* *(0.10)$ \\
\hline Prob F & $\mathrm{C}$ & $* *(0.02)$ & $\star *(1.59)$ & $* *(2.68)$ & $* *(0.84)$ & ${ }^{\star *}(0.10)$ \\
\hline & $\mathrm{A} \times \mathrm{C}$ & $\star *(0.05)$ & $\star *(1.10)$ & $\star *(1.02)$ & $* *(0.06)$ & ${ }^{\star *}(0.12)$ \\
\hline & $V(\%)$ & 4.14 & 2.07 & 1.45 & 1.51 & 2.71 \\
\hline
\end{tabular}

Medias con la misma letra dentro de columnas son estadísticamente iguales (Tukey, 0.05$)$; ns = no significativo $(\mathrm{P} \leq 0.05)$; CS = carbohidratos solubles; FDA = fibra detergente ácida; FDN = fibra detergente neutro; Mont = ambiente de Montecillo; SPI = ambiente de San Pablo Ixayoc; SK = cv. 'Strike'; BV = cv. 'Black Valentine'; H-14 = cv. 'Hav-14'; Prom = promedio; Cifras entre paréntesis representan la diferencia mínima significativa; $\mathrm{CV}=$ coeficiente de variación; $\mathrm{A}=$ ambiente: $\mathrm{C}=$ cultivar; $\mathrm{A} \times \mathrm{C}=$ interacción ambiente $\mathrm{x}$ cultivar. 


\section{AGRADECIMIENTOS}

Al Consejo Nacional de Ciencia y Tecnología (CONACYT), por el apoyo otorgado para la realización de esta investigación. Registro No. 193001 (2007). Al Laboratorio de Nutrición Animal del Departamento de Zootecnia de la Universidad Autónoma Chapingo, por el apoyo para realizar el análisis químico proximal.

\section{BIBLIOGRAFÍA}

Adsule R N, Deshpande S S, Sathe S K (2004) Tratado de Ciencia y Tecnología de las Hortalizas. Ed. Acribia, S.A. México D.F. 739 p.

Cselotei L, G Vargas (1987) The effect of irrigation on the quality and harvest time of snap beans. Acta Hort. 220:337-381.

Doorenbos J, W O Pruitt (1986) Las Necesidades del Agua para los Cultivos. Estudio FAO. Riego y Drenaje. Manual 24. 194 p.

Escalante E J A, J S Kohashi (1993) El Rendimiento y Crecimiento del Frijol. Manual para Toma de Datos. Colegio de Postgraduados. México. $84 \mathrm{p}$.

Esquivel E G, J A Acosta, R Rosales, P Pérez, J M Hernández, R Navarrete, J S Muruaga (2007) Productividad y adaptación del frijol ejotero en el Valle de México. Rev. Chapingo S. Hort. 12:119-126.

M S J Favaro P S, B J A Neto, W H Takahashi, E Miglioranza, I E Ida (2007) Rates of calcium, yield and quality of snap bean. Sci. Agric. 64:616-620.

García E (2005) Modificación al Sistema de Clasificación Climática de KÖppen. 4a ed. UNAM. México, D.F. 217 p.

Guzmán M S H, J A Acosta, M M A Álvarez, D S García, P G Loarca (2002) Calidad alimentaria y potencial nutracéutico del frijol ejotero (Phaseolus vulgaris L.) Agric. Téc. Méx. 28:159-173.

Mawgoud A, M Desuki, S R Salman, A Hussein (2005) Performance of some snap bean varieties as affected by different levels of mineral fertilizers. J. Agron. 4:242-247.

Miglioranza E, R Araujo, M R Endo, P R J Souza, A M Montanari (2003) Teor de cálcio em frutos de diferentes cultivares de feijao-vagem. Hort. Bras.21:158-161.
Peixoto N, L T Braz, D A Banzatto, E A Moraes, F M Moreira (2002) Características agronómicas, productividade, qualidade de vagens e divergencia genetica em Feijoo-vegem de crecimiento indeterminado. Hort. Bras. 20:447-451.Pomper W K, A M Grusak (2004) Calcium uptake and whole-plant water use influence pod calcium concentration in snap bean plants. J. Amer. Soc. Hort. Sci. 129:890-895.

Ríos M J, J M Quirós D (2007) El Frijol su Cultivo, Beneficio y Variedades. Corporación Colombiana de Investigación Agropecuaria. Medellín, Colombia. Manual Técnico. 193 p.

Quintana J M, H C Harrison, J P Palta, J Nienhuis, K Kmiecik (1999) Calcium fertilizers fail to affect pod calcium concentration and yield of four snap bean cultivars. Hort. Sci. 34:646-647.

Rosales S R, M R Ochoa, J A Acosta (2001) Fenología y rendimiento del frijol en el Altiplano de México y su respuesta al fotoperiodo. Agrociencia 35:513-523.

SAGARPA, Secretaría de Agricultura, Ganadería, Desarrollo Rural, Pesca y Alimentación (2009) Producción de hortalizas. Disponible en: http://www.sagarpa.gob.com.mx (Diciembre 2009).

Salinas R N, E J A Escalante, G M T Rodríguez, M E Sosa (2008) Rendimiento y calidad nutrimental de frijol ejotero (Phaseolus vulgaris L.) en fechas de siembra. Rev. Fitotec. Mex. 31:235-241.

Secretaria de Salud (2010) Sobrepeso y obesidad. problema gordo para México. Disponible en http://portal.salud.gob.mx/sites/salud/ descargas/pdf/period_mexsano/mexicosano_ene10.pdf (Junio 2010).

Snyder R L (1985) Hand calculating degree days. Agric. For. Meteor. 35:353-358.

Sosa P E (1979) Manual de Procedimientos Analíticos para Alimentos de Consumo Animal. Departamento de Zootecnia. Universidad Autónoma Chapingo, México. 115 p.

Traka M E, D Georgakis, K M Sotiriou, T Pritsa (2000) An integrated approach of breeding and maintaining an elite cultivar of snap bean. Agron. J. 92:1020-1026.

Yvestirilly C M B (2002) Tecnología de Hortalizas. Ed. ACRIBIA, S.A. Zaragoza, España. 591 p.

Zhiwei Q, X Xangyang, L Hongyu, Y Chengge, T Bing (1995) Evaluation of quality characteristic of the fresh pods of the bean (Phaseolus vulgaris L.) in breeds in Heilongilang province. Acta Hort. 402 200-205. 